\title{
Amiodarone Induced Pulmonary Toxicity: A Fatal Case Report
}

\section{Oyku Gulmez ${ }^{1 *}$ and Aylin Ozsancak ${ }^{2}$}

${ }^{1}$ Department of Cardiology, Baskent University Istanbul Medical and Research Center, Turkey

${ }^{2}$ Department of Pulmonary Medicine, Baskent University Istanbul Medical and Research Center, Turkey

\begin{abstract}
Amiodarone Induced Pulmonary Toxicity (AIPT) is a rare but one of the most serious adverse event that can be potentially life threatening. The incidence of AIPT is $2 \%$ to $10 \%$ and mortality rate ranges from $10 \%$ to $50 \%$ in patients who develop Acute Respiratory Distress Syndrome (ARDS). Age, preexisting lung disease, cumulative dose, thoracic surgery and pulmonary angiography are the risk factors associated with AIPT. Although there is no pathognomonic clinical, laboratory, radiographic or histological findings the diagnosis depends on clinical suspicion and exclusion of other possibilities of pulmonary disease. We present a case of 77 -year-old man who presented with symptoms of pneumonia and diagnosed as AIPT by the elimination method for specific and non-specific pulmonary infection. Despite discontinuation of amiodarone and systemic therapy with corticosteroids the patient continued worsen. Pre-existing lung disease, the rapid onset of the clinical picture and the extent of lung injury on CT were the poor prognostic factors for our patient.
\end{abstract}

Keywords: Amiodarone; Pulmonary toxicity; Thoracic surgery

\section{Introduction}

Amiodarone is an iodinated benzofuran derivate Class III antiarrhythmic drug that is used for effective management and prevention of life-threating ventricular arrhythmias and atrial fibrillation [1,2]. Unfortunately, due to its lipophilic properties, it has a large distribution volume and a wide range of adverse events including pulmonary toxicity, hepatotoxicity, cardiotoxicity, thyroid dysfunction and corneal microdeposits. Of these, amiodaron-induced pulmonary toxicity (AIPT) is a rare but the most important complication of amiodaron due to its non-reversible and fatal potential. The incidence of AIPT is $2 \%$ to $10 \%$ and mortality rate ranges from $10 \%$ to $50 \%$ in patients who develop acute respiratory distress syndrome (ARDS) [3]. Although AIPT has no pathognomonic clinical, laboratory, radiographic or histological findings the diagnosis depends on clinical suspicion and exclusion of other possibilities of pulmonary disease [3,4]. Risk factors for AIPT include age, duration and intensity of therapy (cumulative dose), preexisting lung disease, thoracic surgery and pulmonary angiography $[3,5]$. We describe a case of fatal pulmonary toxicity in a 77-year-old man with known coronary artery disease and preexisting lung disease who presented with progressive dyspnea, cough, weakness and chest imaging and laboratory findings revealed suspicion of pneumonia.

\section{Case Report}

A 77-year-old man was admitted to the hospital with progressive dyspnea, cough and weakness for 1-week duration. He had a medical history of coronary artery disease since 2011 diagnosed by coronary angiography, hypertension, diabetes mellitus, sick sinus syndrome with a definite pacemaker insertion 4 months ago. The patient underwent left upper segmentectomy for giant cell carcinoma 5 years ago (T1ANOM0) and had chemotherapy and radiotherapy. He was maintained on amiodarone $400 \mathrm{mg} /$ daily for the past 2 months due to recurrent episodes of symptomatic atrial fibrillation. His physical examination revealed blood pressure of $120 / 80 \mathrm{mmHg}$, heart rate of 70 beats/min, respiratory rate of 16 breaths $/ \mathrm{min}$, temperature of $37.2^{\circ} \mathrm{C}$ and oxygen saturation of $80 \%$ on room air. Bilateral crackles were heard on examination. At the time of admission, laboratory examination revealed white blood cell count of 9280 bin/uL, 70.1\% neutrophils and $6.2 \%$ eosinophils and elevated C-reactive protein: $142.3 \mathrm{mg} / \mathrm{L}$. Other laboratory workup showed creatinine level of $0.9 \mathrm{mg} / \mathrm{dL}$, brain natriuretic peptide $259.1 \mathrm{pg} / \mathrm{mL}$, thyroid stimulating hormone 0.29 $\mu \mathrm{IU} / \mathrm{mL}$, aspartate aminotransferase $41 \mathrm{U} / \mathrm{L}$, alanine aminotransferase $37 \mathrm{U} / \mathrm{L}$, and electrolyte concentrations within normal limits with no elevation of cardiac enzymes. D-dimer under oxygen level was also within normal limits. Blood gas analysis supply of $5 \mathrm{~L} / \mathrm{min}$ showed hypoxemia without $\mathrm{CO}_{2}$ retention. Urinalysis was unremarkable. Electrocardiography showed sinus rhythm with no ischemic changes. Echocardiographic examination showed normal size and function of left and right ventricle with a left ventricular ejection fraction of $55 \%$. There was also no significant valvular disease. Chest X-ray showed bilateral pulmonary interstitial infiltration (Figure 1). Chest computed tomography demonstrated a diffuse bipulmonary ground glass confluent opacities, and areas of consolidation at the bases resembling ARDS

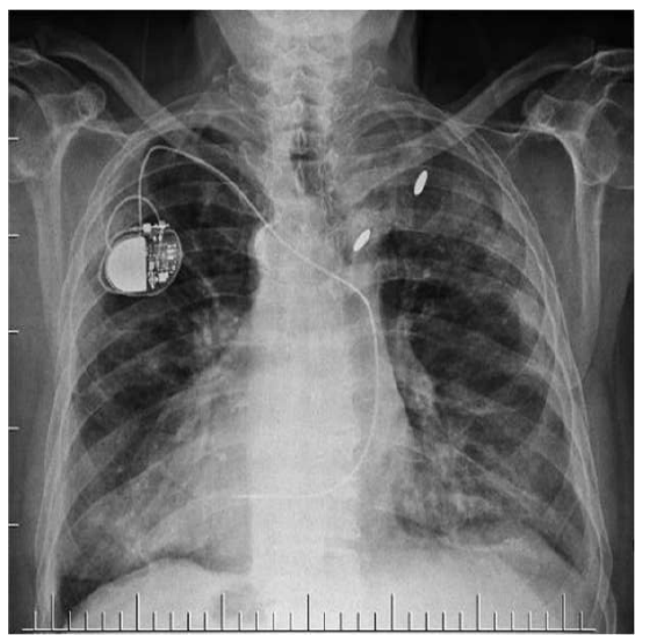

Figure 1: Chest radiograph on admission revealing bilateral infiltrates.

*Corresponding author: Oyku Gulmez, Department of Cardiology, Baskent University Istanbul Medical and Research Center, Turkey, Tel: +90 5352496139; Fax: +902616519858; E-mail: gulmezoyku@yahoo.com

Received July 14, 2017; Accepted July 24, 2017; Published July 31, 2017

Citation: Gulmez O, Ozsancak A (2017) Amiodarone Induced Pulmonary Toxicity: A Fatal Case Report. J Clin Case Rep 7: 998. doi: 10.4172/2165-7920.1000998

Copyright: @ 2017 Gulmez O, et al. This is an open-access article distributed under the terms of the Creative Commons Attribution License, which permits unrestricted use, distribution, and reproduction in any medium, provided the original author and source are credited. 


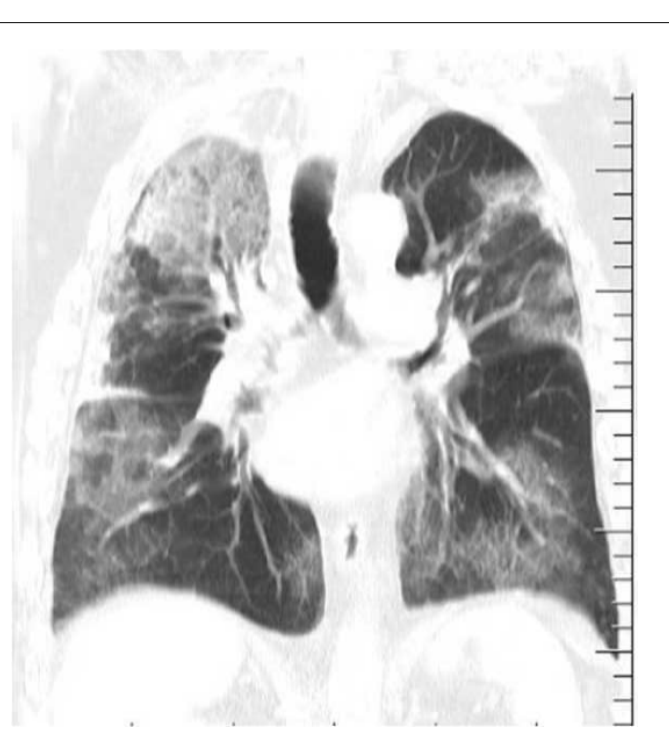

Figure 2: Computed tomographic slices showing patches of ground glass opacity.

(Figure 2). Pulmonary embolism was excluded. Blood cultures were drawn and empiric broad-spectrum antibiotic therapy was initiated. Bronco dilatator therapy was also added to patient's home medications (clopidogrel, apixaban, amlodipine, metoprolol, amiodarone). On day 4, cultures of blood, sputum, and urine were still negative. Procalcitonin was negative. Despite medical therapy on day 5 , patient continued to complain of dyspnea and required noninvasive ventilatory support. On day 6, pulmonary infection was excluded, and based on clinical, laboratory, radiological and microbiological findings, clinical diagnosis of AIPT was made. Amiodarone was discontinued and pulse high dose steroid therapy was administrated. On day 7 , mechanical ventilation was instituted. On day 8, bronchoscopy with Bronchoalveolar Lavage (BAL) was performed after clinical stabilization. BAL revealed intense blood elements, sparse alveolar macrophages and lymphocytes $(71.9 \%$ neutrophiles, $12.7 \%$ lymphocytes, $7.7 \%$ eosinophils). No bacteria or fungi were also identified via microscopic examination or culture of lavage fluid. In addition, screening for pathogenic viruses including influenza strain $\mathrm{H} 1 \mathrm{~N} 1$, influenza A, influenza B, Corona virus OC43229E-NL63-HKU1, parainfluenza 1-2-3-4, rhinovirus, enterovirus, adenovirus, respiratory syncytial virus $\mathrm{A}, \mathrm{B}$, human matepneumo virus and bocavirus were negative. Additionally, tests for antiviral antibodies (hepatitis $B$ virus, hepatitis $C$ virus, human immunodeficiency virus, cytomegola virus) were negative. Moreover, serological (Legionnella, Chlamydophia pneumonia, Mycoplasma pneumonia, Bordetella pertusis) and immunological tests (Ig A, Ig G, and Ig M) were normal. Galactomannan level was within normal levels. He improved symptomatically and radiographically within 4 days. On day 15 , he was extubated but he was still requiring $5 \mathrm{~L}$ to $8 \mathrm{~L}$ intranasal oxygen. On day 18 , he again presented progressive dyspnea with high oxygen requirements and again intubated. However, the patient died after 12 hours in respiratory failure.

\section{Discussion}

In this case we report a fatal case of amiodarone pulmonary toxicity. Initial treatment included antibiotics because pneumonia was suspected. The AIPT diagnosis was made based on clinical risk factors for AIPT and sterile culture results at a median of 6 days post-admission. Risk factors of our patient were age, pre-existing lung disease and a daily dose of
$400 \mathrm{mg}$ amiodarone. Unfortunately, our patient did not benefit from discontinuation of amiodarone, and steroid therapy and continued worsen. Pre-existing lung disease, the rapid onset of the clinical picture and the extent of lung injury on CT were the poor prognostic factors for our patient.

Despite various adverse events, amiodarone is a highly effective antiarrhythmic agent that is used for both ventricular and supraventricular arrthymias. For supraventricular arrthymias maintenance doses are limited to $200 \mathrm{mg} / \mathrm{d}$ to $400 \mathrm{mg} / \mathrm{d}$ [6,7]. AIPT was first reported in 1980 [8]. Although the incidence of AIPT is declined due to learning curve in maintenance doses (reduced doses) and diagnosing AIPT, it is reported to be $5 \%$ to $10 \%$ [9].

The mechanism of AIPT is incompletely understood but direct toxic effects of the accumulated metabolite of the drug in the pulmonary tissue, $\mathrm{T}$-cell which are predominately CD8 positive mediated immunologic reactions in genetically predisposed patients and the activation of angiotensin enzyme system are the possible mechanisms of AIPT. The high volume of drug distribution close to 5000 liters, extensive tissue binding and long elimination half-life of approximately 30 days to 108 days may explain the toxic effects of the drug seen at any time during treatment, and even months after discontinuation of therapy [3]. Toxicity can present either in acutely in the form of fever, pleuritic chest pain, and cough or sub acutely with chronic non-productive cough, progressive dyspnea, low-grade fever, malaise, weight loss and hypoxia [3]. Moreover, there is no threshold to avoid toxicity from amiodarone [3].

The risk factors for AIPT are age, duration and intensity of amiodarone therapy, pre-existing lung disease, pulmonary angiography, cardiothoracic surgery and high concentrations of oxygen. It is shown that there is positive correlation between age and AIPT. AIPT increases 3 -fold for every 10 years of age in patients aged $>60$ years compared to those aged $<60$ years. Patients receiving a daily dose greater than $400 \mathrm{mg} /$ day are at higher risk compared to those receiving $200 \mathrm{mg} /$ day $[10,11]$. Moreover, a study among Japanese patients demonstrated that the incidence of AIPT increased from $4.2 \%$ to $7.8 \%$ and $10.6 \%$ with 1.3 years and 5 years use of amiodarone respectively, with a mean maintenance dose of $141 \mathrm{mg}$ daily. Additionaly, AIPT is positively correlated with a cumulative dose of $140 \mathrm{~g}$ to $230 \mathrm{~g}$ [12]. In several studies pre-existing pulmonary disease was associated with higher risk of AIPT and AIPT occurred in 50\% surgical survivors, and in $27 \%$ pneumonectomy patients [13-15].

Clinical symptoms, signs, laboratory and radiographic findings of AIPT are nonspecific and there is no clinical, physiological, biological or radiological gold standard to diagnose AIPT. The clinical presentations of AIPT include chronic non-specific interstitial pneumonitis, chronic eosinophilic pneumonia, organizing pneumonia, idiopathic pulmonary fibrosis, desquamate interstitial pneumonia, solitary pulmonary mass, diffuse alveolar hemorrhage and ARDS. Pleural effusion which is usually unilateral and at the right side, or bilateral, can be seen during amiodarone treatment mostly after 6 months of therapy. Pleural effusion can also be seen as early as 2 months and as late as 6 years after treatment [16-18]. Moreover, AIPT may mimic other diseases like congestive heart failure and pulmonary embolism. Due to its nonspecific nature and no definite diagnostic criteria, AIPT is a diagnosis of exclusion of other possible diseases.

Several studies, except one showed that amiodarone is a safe antiarrhythmic agent after lung resection [19-21]. Only one study by Mieghe et al. showed increased risk of ARDS, especially in 
pneumonectomy patients. However, in a recent study by Teerakanok et al. the incidence of amiodarone induced postoperative (mostly after cardiothoracic surgery) ARDS was reported to be $15 \%$ [22]. The possible mechanisms are high oxygen administration, lung damage of intubation/ventilation, the systemic inflammatory response induced by surgery. As one of the mechanism of AIPT is the production of toxic oxygen radicals which can cause direct cellular injury, patients with pre-existing lung disease and lung insult from surgery may be prone to amiodarone toxicity. Nevertheless, patients in whom ARDS develops in the context of pulmonary toxicity related to amiodarone show a fatal course despite therapies.

The primary treatment of AIPT is the suspicion of AIPT after exclusion of other possible pulmonary diseases. Discontinuation of amiodarone is the cornerstone of the treatment. However, due to its accumulation in lung tissue and long elimination half-life time pulmonary toxicity may progress despite drug discontinuation. Systemic corticosteroids ( $40 \mathrm{mg}$ to $60 \mathrm{mg}$ prednisolone per day) are recommended for earlier recovery and decreased parenchymal fibrosis, for at least 4 months to 12 months to avoid relapse [3,23]. Slow improvement as long as three to six months can be noted. Permanent pulmonary fibrosis and death can be seen in case of delayed treatment or refractory cases. Mortality rate is $10 \%$ in patients who presented with AIPT, but may be as high as $20 \%$ to $30 \%$ in patients who require hospital admission, and 50\% in patients who develop ARDS [3].

\section{Conclusion}

Amiodarone toxicity which may occur even with small doses and short treatment duration should be taken into consideration, especially in elderly patients with underlying lung disease who presents with shortness of breath. Early recognition of AIPT is critical to prevent or minimize its potentially pulmonary effects. Amiodarone-induced lung diseases usually have good prognosis when treated and diagnosed early. However, patients who develop ARDS or pulmonary fibrosis have worse prognosis. Patients education about signs and symptoms and regular clinical follow-up are essential for timely diagnosis and the treatment.

\section{References}

1. ESC guidelines for the management of atrial fibrillation developed in collaboration with EACTS (2016) The task force for the management of atrial fibrillation of the European Society of Cardiology (ESC) developed with the special contribution of the European Heart Rhythm Association (EHRA) of the ESC. Endorsed by the European Stroke Organization (ESO) Eur Heart J37: 2893-2962

2. ESC guidelines for the management of patients with ventricular arrhythmias and the prevention of sudden cardiac death (2015) The task force for the management of patients with ventricular arrhythmias and the prevention of sudden cardiac death of the European Society of Cardiology (ESC). Endorsed by Association for the European Paediatric and Congenital Cardiology. Eur Heart J 36: 2793-2867

3. Papiris SA, Triantafillidou C, Kolilekas L, Markoulaki D, Manali ED (2010) Amiodarone: Review of pulmonary effects and toxicity. Drug Saf 33: 539-558.

4. Benassi F, Molardi A, Righi E, Santaggelo R, Meli M (2015) ECMO for pulmonary rescue in adult with amiodarone-induced toxicity. Heart vessels 30 : 410-415

5. Fadahunsi O, Krol R (2014) Acute amiodaron pulmonary toxicity following lung resection. Int J Biomed 10: 217-220

6. Mankikian J, Favelle O, Guillon A, Guilleminault L, Cormier B, et al. (2014)
Initial characteristics and outcome of hospitalized patients with amiodarone pulmonary toxicity. Resp Med 108: 638-646.

7. Camm J, Lüsher TF, Serruys PW (2009) The ESC texbook of cardiovascular medicine (2nd edn). New York: Oxford University Press, USA.

8. Rotmensch HH, Liron M, Tupilski M, Laniado S (1980) Possible association of pneumonitis with amiodarone theraphy. Am Heart J 100: 412-413.

9. Dusman RE, Stanton MS, Miles WM, Klein LS, Zipes DP (1990) Clinica features of amiodaron-induced pulmonary toxicity. Circulation 82: 51-59.

10. Yamada Y, Shiga T, Matsuda N, Hagiwara N, Kasanuki H (2007) Incidence and predictors of pulmonary toxicity in Japanese patients receiving low-dose amiodarone. Circ J 71: 1610-1616.

11. Ott MC, Khoor A, Leventhal JP, Paterick TE, Burger CD (2003) Pulmonary toxicity in patients receiving low-dose amiodarone. Chest 123: 646-651.

12. Cheng HC, Wang JH, Wang ML, Sung MT, Lin SL, et al. (2010) Adverse effect of low-dose amiodarone mimicking pulmonary malignancy. Int $\mathrm{J}$ Angiol 19: e51-e53.

13. Olshansky B, Sami M, Rubin A, Kostis J, Shorofsky S, et al. (2005) Use of amiadorone for atrial fibrillation in patients with preexisting pulmonary disease in the AFFIRM study. NHLBI AFFIRM investigators. Am J Cardiol 95: 404-405.

14. Greenspon A, Kidwell G, Hurley W, Mannion J (1991) Amiodarone-related postoperative adult respiratory distress syndrome. Circulation 84: III407-III415.

15. Van Mieghem W, Coolen L, Malysse I, Lacquet L, Deneffe G, et al. (1994) Amiodarone and the development of ARDS after lung surgery. Chest 105 1642-1645.

16. Hawatmeh A, Thawabi M, Jmeian A, Shaaban H, Shamoon F (2017) Amiodarone-induced loculated pleural effusion without pulmonary parenchymal involvement: A case report and literature review. J Nat Sci Biol Med 8: 130-133.

17. Clarke B, Ward DE, Honey M (1985) Pneumonitis with pleural and pericardial effusion and neuropathy during amiodarene therapy. Int J Cardiol 8: 81-88.

18. Carmichael LC, Newman JH (1996) Lymhocytic pleural exudate in a patient receiving amiodarone. $\mathrm{Br} \mathrm{J}$ Clin Pract 50: 228-230.

19. Berry MF, D'Amico TA, Onaitis MW (2014) Use of amiodarone after major lung resection. Ann Thorac Surg 98: 1199-1206.

20. Barbetakis N, Vassiliadis M (2004) Is amiodarone a safe antiarrhythmic to use in supraventcicular tachyarrhythmias after lung cancer surgery? BMJ Surg 4: 7.

21. Ciriaco $P$, Mazzone $P$, Canneto $B$, Zannini $P(2000)$ Supraventricular arrhythmia following lung resection for non-small cell lung cancer and its treatment with amiodarone. Eur J Cardiothrac Surg 18: 12-16.

22. Teerakanok J, Tantrachoti P, Chariyawong P, Nugent K (2016) Acute amiodarone pulmonary toxicity after surgical procedures. Am J Med Sci 352: 646-651.

23. Goldschlager N, Epstein AE, Naccarelli GV, Olshansky B, Singh B, et al. (2007) A practical guide for clinicians who treat patients with amiodarone. Heart Rhythm 4: 1250-1259. 